\title{
QUELQUES APERÇUS SUR LES PROLÉGOMÈNES A LA PHILOSOPHIE DE DAVID L'INVINCIBLE
}

David l'Invincible, commentateur et philosophe arménien de l'École néoplatonicienne d'Alexandrie, est situé par certains philologues au $\mathrm{V}^{\mathrm{e}}$ et par d'autres au VI ${ }^{\mathrm{e}}$ siècle. En effet sa chronologie est encore souvent objet de discussion. Il est considéré comme l'introducteur du savoir philosophique systématique en Arménie, étant donné que la traduction et diffusion de ses œuvres y permit la classification et systématisation du savoir et des sciences. Nous voudrions introduire la figure et la pensée de David l'Invincible, en sollicitant à la fois des questions fondamentales qui attendent encore des réponse. Certaines des questions font l'objet d'une recherche actuelle dans laquelle nous sommes engagée.

Après la création de l'alphabet par Mesrop Maštoc', ses disciples ouvrirent une époque d'activité de traduction, qui aurait perdurée jusqu'à VII-VIII ${ }^{\text {ème }}$ siècles. L'intérêt des premiers traducteurs ${ }^{1}$ concerna la littérature patristique, en particulier celle à caractère liturgique, homilétique, exégétique et apologétique. Quant aux traducteurs postérieurs, nommés traducteurs de la deuxième génération, ils furent plus intéressés par la traduction des œuvres philosophiques grecques. Les auteurs traduits furent les philosophes grecs et hellénistiques entre autre: Porphyre, Philon l'Hébreu d'Alexandrie, Jamblique et Olympiodore. La floraison de la littérature arménienne commença à partir du V $V^{\text {ème }}$ siècle, connu comme le siècle d'or de la littérature et de la langue, grâce à l'invention de l'alphabet. D'ailleurs son inventeur eut l'intuition de forger l'identité de la nation arménienne ${ }^{2}$ par la création de l'alphabet et d'une culture littéraire à travers la diffusion des textes traduits, surtout de la langue grecque.

${ }^{1}$ Le cercle des premiers traducteur était constitué par Mesrop lui-même, le Catholicos Sahak Partew et leur disciples directs, Eznik de Kołb, Koriwn vardapet, Yovsēp' Pałanakan et Yovhan Ekełec'ac'i. Une des premières traduction de la Bible fut celle des Proverbes, réalisée par Mesrop, et ensuite celles de tout le texte sacré.

${ }^{2}$ Cfr. B.L. Zekiyan, L'idéologie nationale de Movses Xorenac'i et sa conception de l'Histoire, „Handēs Amsorya” 101 (1987) 471-485; idem, L'identité polyvalente dans la témoignage d'un artiste: Serguei Paradjanov, ,Acta Orientalia Academiae Scientiarum Hungaricarum” 50 (1997) 337-347. 
La figure de David l'Invincible a été toujours entouré d'une auréole légendaire, en tant que la plupart des sources de la tradition arménienne concordent à reconnaître en David un des disciples directs de Mesrop Maštoc'. Cependant le style hellénisant de ses textes confirme la leur rédaction par le cercle des traducteurs de la deuxième génération. D'ailleurs les sources de la tradition arménienne distinguent deux générations des traducteurs et relient à la deuxième la production des œuvres de l'Ecole hellénisante. En plus d'autres sources le présente comme un condisciple du père de l'histoire arménienne, Moïse de Chorène, qu'on peut placer au dernier quart du $\mathrm{V}^{\mathrm{e}}$ siècle $^{3}$. Plusieurs textes sur arguments différents, philosophiques et théologiques, lui sont attribués; ainsi on compte presque 25 æuvres originales et 26 traductions dans l'édition de 1833 des Pères Mekhitaristes de Saint Lazare à Venise. On peut distinguer au moins six auteurs, postérieurs à David lui-même, dont les œuvres ont été attribuées à I'Invincible, tandis que les œuvres, qui appartient indéniablement à David, forment le Corpus Davidicum, constitué de quatre textes: les Définitions et Divisions de la philosophie, le Commentaire à l'Isagoge de Porphyre, le Commentaire aux Catégories d'Aristote et le Commentaire aux Analytiques d'Aristote. Pour ce qui concerne le Commentaire aux Catégories il y a deux opinions entre les savants; certains soutiennent qu'il faut l'attribuer à Elias (fin du $\mathrm{V}^{\mathrm{e}}$ - première moitié du $\mathrm{VI}^{\mathrm{e}}$ siècle) ${ }^{4}$ et d'autres à David. Entre ces deux points de vue, celle qui nous paraît s'appuyer sur des preuves plus convaincantes, est celle qui attribue la paternité du texte à David.

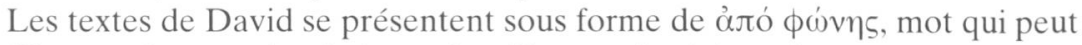
signifier que le texte fut écrit par les élèves qui suivirent les cours du maître:

Cfr. St. Malxaseanc', Xorenac'u aŕetcvaci šurja (Sur l'énigme de Xorenac'i), Erevan 1940; G. Traina, Il complesso di Trimalcione - Movsēs Xorenac'i e le origini del pensiero storico armeno, Venezia 1991.

${ }^{4}$ La figure d'Elias appartient au cercle d'Olympiodore le Mineur, probablement chef de l'école d'Alexandrie du début du VI ${ }^{\text {ème }}$ siècle à la deuxième moitié du VI ${ }^{\text {mème }}$ siècle. Selon l'opinion de la plus part des savants Elias et David étaient disciples d'Olympiodore ou moins ses contemporains, parce que il y a beaucoup des ressemblances entre eux pour ce qui concerne le moyen de développer les argumentations dialectiques dans leur textes. Néanmoins il y a aussi une ressemblance étroite entre David et Ammonius, qui avait enseigné du début à la deuxième moitié du $\mathrm{V}^{\mathrm{ème}}$ siècle. Le premier savant qui a refusé la paternité du Commentaire aux Catégories à David a été Adolf Busse, l'éditeur de la version grecque (Commentaria in Aristotelem Graeca), en appuyant sa thèse sur trois preuves textuelles. Sa thèse a été réfutée point à point par Sen Arevšatyan, l'éditeur de la version critique arménienne et de la traduction de quatre textes attribués à David en langue arménienne moderne et russe, cfr. S. Arevšatyan, Dawit' Anhatt $i$ žarangut'yund nor lusabanut yamb [L'héritage de David l'Invincible sous une nouvelle lumière], „Banber Matenadarani” 9 (1969) 7-22; J.P. Mahé, David l'Invincible dans la tradition arménienne, dans: Philosophia Antiqua, Simplicius-Commentaire sur les Catégories, éd. I. Hadot, fasc. I, Leiden 1989, 189-207. 
c'est-à-dire que l'enseignent donnait ses leçons et les élèves prenaient des notes qui auraient constitué le texte écrit. Néanmoins, lors de l'époque byzantine la locution pouvait indiquer la paternité de l'auteur, en signifiant „écrit par”, donc en ce cas „écrit par David”.

Le curriculum studiorum suivi par les étudiants de l'École d'Alexandrie était commun à tous les étudiants des Académies néoplatoniciennes de l'Antiquité, dans lesquelles l'étude de la pensée de Platon jouait un rôle dominant. Donc l'élève commençait à aborder l'étude de la logique d'Aristote, considérée indispensable à la compréhension des systèmes philosophiques non seulement d'Aristote, mais aussi, et surtout, de Platon. Avant l'étude de la logique il était nécessaire d'introduire les arguments dialectiques par la lecture et l'explication de l'Isagoge de Porphyre, que l'on peut considérer comme une introduction non seulement aux Catégories d'Aristote, mais aussi à la méthode dialectique et, finalement, à la logique. Cependant l'Isagoge a servi aussi d'introduction à la philosophie en général: avant sa lecture, les parcours des études alexandrines prévoyaient une introduction à la philosophie, de laquelle nous sont parvenus trois textes différents sous forme des Prolégomènes à la philosophie. Ces textes sont notamment ceux d'Ammonius, d'Elias et de David. Après la lecture et l'apprentissage de l'Isagoge, il fallait que l'élève abordât la pensée d'Aristote en commençant par la lecture de l'Organon et en poursuivant avec des traités concernant l'éthique, la physique, les mathématiques et, enfin, la métaphysique. L'argument de l'Organon était introduit d'un Commentaire aux Catégo-

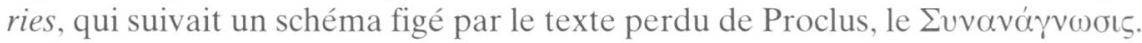
Le texte se développait en dix points argumentatifs, selon la témoignage de David lui-même. Quant aux Prolégomènes des Commentaires aux Catégories, nous sont parvenus ceux d'Ammonius, de Jean Philopone, d'Olympiodore, de Simplicius et de David (Elias), qui présentent tous des ressemblances frappantes. Le commentaire et la lecture du traité d'Aristote suivaient aux prolégomènes. En outre, on a conservés les Commentaire au De Interpretatione et le Commentaire aux Analytiques Premiers.

Nous avons un témoignage intéressant d'un auteur musulman du $\mathrm{X}^{\text {ème }}$ siècle, al-Miskawayh, qui dans un des ses traités, le Tartib as-sa'ādāt (Le règlement des bonheurs), donne une idée sur le parcours des études d'Aristote et de Platon à l'école d'Alexandrie. L'auteur mentionne la classification des parties de la philosophie chez Aristote, en s'appuyant sur le texte de Paule le Perse $^{5}$, l'Introduction à la logique, dont une partie est conservée seulement

${ }^{5}$ Paul le Perse était un philosophe et théologien nestorien, qui travaillait à la court de Khusraw Anūširwān (531-568), le souverain illuminé qui avait donné hospitalité aux philosophes grecs obligés à la fuite d'Athènes après la fermeture de l'Académie (529) par l'empereur Justinien, cfr. D. Gutas, Paul the Persian on the classification of the parts of Aristotle's philosophy: a milestone between Alexandria and Bagdād, „Der Islam” 60 (1983) 231-267 ou dans: D. Gutas, Greek Philosophers in the Arabic Tradition (variorum reprints), London 2000, 231-267. 
chez al-Miskawayh. La classification mentionnée par Paul le Perse et, successivement, par al-Miskawayh est la même que l'on trouve dans les Prolégomènes des Commentaires aux Catégories chez les auteurs alexandrins. Selon Paule, le temps nécessaire à un étudiant pour apprendre Aristote était un minimum de dix ans et un maximum de vingt ans, et, enfin, après l'étude d'Aristote, il suivait la lecture de Platon, considéré le sommet du savoir philosophique. Comme il faillait une préparation propédeutique pour aborder la philosophie d'Aristote, une introduction à la pensée de Platon était nécessaire. Le seul exemple des Prolégomènes à la philosophie de Platon a été retrouvé et publié par L.G. Westerink ${ }^{6}$, mais la paternité du texte reste encore anonyme. Selon le Westerink, on peut ramener ce traité à un traité perdu d'introduction à la philosophie platonicien de Proclus. L'auteur anonyme du texte se rappelle à l'autorité de Proclus et Jamblique et en tire le règlement de lecture des douze dialogues platoniciens: l'Alcibiade Premier, le Gorge, le Phédon, le Cratyle, le Théétète, le Sophiste, le Politique, le Phèdre, le Symposium, le Philèbe, le Timée et enfin le Parménide ${ }^{7}$.

Les commentaires alexandrins se partagent selon un schéma lié aux exigences d'enseignement de l'école. Partant le traité est partagé en práxeis, c'està-dire en leçons, qui s'étendaient sur toute la journée et pendant lesquelles l'enseignant développait un thème particulier. En effet les leçons se partageaient, à leur tour, en deux sections: théorie $(\theta \varepsilon \omega \varrho i \alpha)$ et expression $\left(\lambda \varepsilon^{\prime} \xi ı\right)^{8}$. Dans la première partie le professeur expliquait synthétiquement le texte, dont la lecture était faite ensemble au début de la leçon, tandis que dans la deuxième partie il donnait une exégèse approfondie du texte lu. En général, les traités présentent une division exprimée directement en théories, la section de $\lambda \dot{\varepsilon} \xi ı \zeta$ y étant souvent sous-entendue. Nous trouvons cette division dans les Commentaires à l'Isagoge, dans les Commentaires aux Catégories et aux autres traités d'Aristote, tandis que les Prolégomènes à la philosophie de David s'en

${ }^{6}$ Cfr. L.G. Westerink, Anonymus Prolegomena to Platonic Philosophy, Amsterdam 1962. La paternité du traité est anonyme, mais selon le savant l'époque de production de l'œuvre est très probablement le $\mathrm{VI}^{\mathrm{I}}{ }^{\mathrm{e}}$ siècle et, le milieu, celui d'Alexandrie. Le traité se développe de la façon suivante: biographie de Platon, introduction à la philosophie de Platon (de quel genre est-elle, pourquoi Platon a-t-il retenu nécessaire écrire sa pensée, etc.), introduction aux douze dialogues.

${ }^{7}$ La tradition arabe nous apporte un témoignage précieux chez l'historien al-Mas'ūdì (m. 957) qui, dans une de ses œuvres, raconte sa visite à l'école des Sabiens de Harrān et dit que sur le montant de la porte à l'entrée de l'école il y avait gravé un discours tiré par l'Alcibiade Premier de Platon. Donc les Sabiens de Harrān faisaient philosophie en suivant l'impératif d'enseignement figé par Olympiodore, selon lequel l'Alcibiade était le seuil du temple dont le centre était le Parménide, cfr. M. Tardieu, Sābiens coraniques et «Sābiens» d'Harrān», „Journal Asiatique” 274 (1986) 1-44.

${ }^{8}$ Cfr. R. Vancourt, Les derniers commentateurs alexandrins d'Aristote. L'école d'Olympiodore, Étienne d'Alexandrie, Thèse complémentaire présentée pour le doctorat des Lettres, Lille 1941 
distinguent. En effet, dans les Prolégomènes, le texte se divise en praxeis/leçons qui coïncident, en fait, avec les exposés théroiques ( $\theta \varepsilon \omega \varrho i \alpha \iota$ et $\lambda \dot{\varepsilon} \xi \varepsilon ı$ ), pendant lesquels le professeur présentait le sujet de la leçon d'une façon approfondie et accomplie.

Un témoignage sur la méthodologie suivie dans les écoles de l'Antiquité, en particulier en Alexandrie, se trouve chez le traducteur arabe Hunayn ibn Isḥāq, qui décrit le méthode d'enseignement des professeurs de médecine d'Alexandrie et celle adoptée par les écoles de la même période. Il dit que les élèves se rassemblaient tous les jours à lire les œuvres de Galen, tandis que d'autres textes étaient lus individuellement. La méthode d'enseignement suivie par les élèves des écoles de médecine aurait été appliquée dans les autres disciplines, comme la logique et la philosophie.

Comme nous avons déjà dit, nous avons trois versions des Prolégomènes à la philosophie: celle d'Ammonius, de David et d'Elias. La structure textuelle de trois versions ne présente pas des différences frappantes, il y a plutôt des différences d'exposition de la matière que nous essayerons de peindre à partir du texte de David. Les Prolégomènes de David se composent de vingt $\pi \varrho a ́ \xi \varepsilon ı \zeta$ dans la version grecque et vingt et un dans la version arménienne, tandis que le texte d'Elias se structure en douze chapitres.

Le texte de David se présente de la façon suivante: au début, l'auteur aborde la question de l'ens en l'articulant en quatre interrogations: existe-t-il, qu'est-il, quel est-il et pourquoi est-il. Cette argumentation se trouve chez Elias, mais pas chez Ammonius. Donc il aborde la question de l'existence de la philosophie à travers la discussion et la réfutation de quatre argumentations des Sceptiques, qui nient la possibilité de connaître l'être, soumis à l'homonymie et à un procès de changement perpétuel, et il est donc impossible de faire des enquêtes sur l'être, qui est le soubassement de la philosophie. Après avoir réfuté les argumentations sceptiques en évoquant l'autorité de Platon et Aristote, David conclut cette section de la façon suivante:

„Donc, s'il ya Dieu, il y a aussi la providence, puisque Dieu ne crée pas seulement les créatures, mais il a aussi soin d'eux. Alors si la providence existe, il y a aussi l'intellect, puisque il n'a pas soin de quelque chose sans raison, mais avec intelligence. Et s'il y a l'intellect, il y aussi le désir d'intellect. Et s'il y a le désir d'intellect, il y aussi l'amour d'intellect, et s'il y a l'amour d'intellect, alors il y a aussi la philosophie, puisque quoi d'autre est l'amour d'intellect que la philosophie" .

${ }^{9}$ Dawit' Anyałt', Erkasirut'iwnk' p'ilisop'ayakank' (ch. I) [Euvres philosophiques], éd par S. Arevšatyan, Erevan 1980, 37. 
Par la suite David ouvre la question sur la substance de la philosophie, que l'on peut connaître soit par la définition comme une unité et soit par la subdivision comme une multiplicité. Pour ce qui est de la définition de la philosophie, il y a neuf questions préliminaires, qui concernent notamment l'état ontologique de la définition ${ }^{10}$ : la différence entre la description et la définition descriptive ${ }^{11}$, l'origine du $\operatorname{mot}^{12}$, de quoi se forme la définition ${ }^{13}$, les définitions parfaites et imparfaites ${ }^{14}$, les six définitions de la philosophie ${ }^{15}$, la démonstration de la raison pour laquelle les définitions sont six ni plus ni moins. Cette démonstration est fournie au moyen de la division et de l'arithmétique; d'abord, grâce à la division on démontre qu'il y a, en premier lieu, deux définitions qui viennent l'une de l'étymologie et l'autre du primat d'excellence, et après il y a quatre définitions qui viennent du fait que la philosophie a un sujet proche et lointain et une fin proche et lointain ${ }^{16}$. D'autre côté, au

${ }^{10}$ Cfr. ibidem (ch. III), p. 40-41: ,,a définition est un discours bref, qui révèle la nature du sujet en question. [...] Et il faut savoir que l'homme est un animal raisonnable, mortel, réceptif d'intellect et de pensée. Donc à la place du genre on considère l'animal, qui exprime un concept général et valable pour plusieurs sujets; en effet il est valable pour l'homme, le cheval, le chien et des autres sujets semblables, tandis que les autres mots sont à la place des différences spécifiques”.

${ }^{11}$ Cfr. ibidem (ch. III), p. 43: „Il faut savoir que la définition se distingue de la description pour la raison que la première prend des voix essentielles et révèle l'essence et la nature du sujet en question, par exemple: homme, animal raisonnable, mortel, réceptif d'intellect et de pensée. D'autre coté la description prend des accidents et révèle les circonstances du sujet en question, par exemple: l'homme est bien droit, riant et a les ongles larges".

12 Cfr. ibidem (ch. IV), p. 45: „Il faut savoir qu'on parle de définition à partir du déplacement des frontières des villages et des champs, parce que les ancêtres, en fuyant de l'excès et de la pénurie, inventèrent la frontière [...]. Donc la définition circonscrit le sujet et le distingue des autres, comme par exemple la définition d'homme, qui dit animal rational, mortel, réceptif d' intellect et de science. Donc quand elle dit animal elle le distingue de ceux qui ne sont pas animal, c'est-à-dire les non-animaux et les inanimés; quand elle dit raisonnable, elle le distingue des irraisonnables, et quand elle dit mortel, elle le distingue des immortels".

13 Cfr. ibidem (ch. IV), p. 46: „Et il faut savoir que les définitions se constituent du sujet, du fin ou de tous les deux, c'est-à-dire du sujet et du fin. Mais d'abord nous dirons ce qu'est le sujet et ce qu'est le fin. Le sujet est ceci selon quoi l'art et la science agissent, tandis que le fin est ceci selon quoi elles sont finalisées, se configurent et constituent le tout [...]. En effet la philosophie a le sujet et le fin. Le sujet sont tous les êtres, tandis que le fin est la science d'eux, c'est-à-dire de les connaître et de devenir semblables à Dieu par moyen de leur connaissance".

${ }^{14}$ Cfr. ibidem (ch. IV), p. 47: „Il faut savoir que la définition parfaite est celle qui se convertit au sujet à définir, [...] tandis que la définition imparfaite est celle qui ne se convertit pas, puisque le fait qu'elle ne se convertit pas exprime la négativité de la définition”.

${ }^{15}$ Cfr. ibidem (ch. V), p. 52: „D'abord la philosophie est la science des êtres tels quels ils sont. La deuxième dit que la philosophie est la science des choses divines et humaines. La troisième dit que la philosophie est méditation de la mort. La quatrième dit que la philosophie est la ressemblance de Dieu selon la possibilité humaine. La cinquième dit que la philosophie est l'art des arts et la science des sciences. La sixième dit que la philosophie est l'amour de la sagesse".

${ }^{16}$ Cfr. ibidem (ch. V), p. 53: „De même façon la philosophie a ainsi le sujet prochain et lointain, le fin prochain et lointain. Donc comme sujet prochain elle considère les êtres en géné- 
moyen de l'arithmétique on démontre qu'il y a des nombres parfaits, imparfaits et ultra-parfaits; les nombres parfaits, dans lesquels il y a le six, sont ceux dont les parties sommées sont égales au nombre entier; les imparfaits sont ceux dont les parties sommées sont insuffisantes par rapport à l'entier; et, enfin, les ultraparfaits sont ceux dont les parties sommées sont excédantes par rapport à l'entier. Le premier nombre parfait est le six, donc les définitions de la philosophie sont six, tandis que les divisions des définitions de la philosophie sont quatre. En effet, le quatre était considéré par les Pythagoriciens comme le nombre pur, puisque en l'additionnant aux nombres qui le précèdent, il donne dix, c'est-à-dire le quatre additionné à trois donne sept, qui additionné à deux donne neuf, qui additionné enfin à un donne dix. Et dix est un nombre parfait, parce que jusqu'à dix les unités ont des noms propres, tandis qu'elles se rattachent au dix pour former les nombres suivants dix. En outre il y a aussi le nombre vierge, sept, comme il est défini par David. En tant que nombre vierge, sept ne génère pas un nombre et ne se génère pas d'un nombre au-dessous de dix, par addition ou par multiplication. Par contre, les autres unités jusqu'à dix sont générées de nombres au-dessous de dix et génèrent des nombres audessous de dix, par addition ou par multiplication.

La huitième question sur l'état ontologique de la définition concerne l'ordre des six définitions, selon lequel les dernières parmi les définitions sont celles qui viennent de l'étymologie et de l'excellence, tandis que les premières et les plus importantes sont celles qui sont liées au sujet et à la fin. Entre cellesci, les premières sont les définitions qui viennent du sujet proche, parce que le sujet est plus important que la fin et le sujet proche est plus important que le sujet lointain.

La neuvième question concerne les créateurs de ces définitions, notamment les définitions suivantes: „la philosophie est la science des êtres tels qu'ils sont”, „la philosophie est la science des choses divines et humaines”, ,la philosophie est être amoureux de la sagesse" appartiennent à Pythagore, tandis que les définitions ,la philosophie est méditation sur la mort", ,la philosophie est ressemblance à Dieu selon les humaines possibilités" appartiennent à Platon et, enfin, la définition ,la philosophie est l'art des arts et la science des sciences" appartient à Aristote.

Ensuite David passe à expliquer la division de la philosophie en philosophie théorie et pratique; mais avant de l'exposer il veut donner la différence entre les

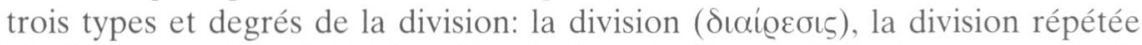

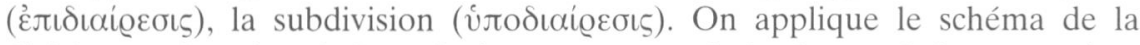
division au domaine de l'ontologie, notamment de la chose subsistante, qui en

ral, tandis que comme sujet lointain a les choses divines et humaines. Encore, comme fin prochain elle a la méditation sur la mort, c'est-à-dire la mort des passions, tandis que comme fin lointain elle a, par la mort des passions, la ressemblance de Dieu selon la possibilité humaine". 
se divisant devient plus particulière et définie, par exemple animal. D'abord on sépare, selon la division, l'animal en rationnel et irrationnel; ensuite on divise de nouveau l'animal rationnel en mortel et en immortel et, enfin, on divise le mortel en homme et cheval et l'immortel en ange et démon. La philosophie suit le même schéma, en se partagent en philosophie théorique et pratique, qui à la fois se divisent de manières différentes selon les Platoniciens et les Aristotéliciens. En effet, selon les premiers, la théorie se divise en physique et en théologie, tandis que les mathématiques sont propédeutiques à la philosophie. Les Aristotéliciens considèrent les mathématiques partie de la théorie. David accepte la division d'Aristote et justifie la division en trois parties pour la raison que les êtres aussi se manifestent selon trois formes et la connaissance de cellesci est liée aux trois disciplines de la philosophie théorique. Á ce point, David expose ainsi sa théorie gnoséologique: la connaissance de tous les êtres est le sujet de la philosophie théorique, et les êtres peuvent se manifester matériellement dans la subsistance et dans la pensée, comme le bois ou la pierre; matériellement dans la subsistance et immatériellement dans la pensée, comme les figures géométriques; immatériellement dans la subsistance et la pensée, comme Dieu, les anges, la pensée et l'âme. Donc il y a trois degrés de connaissance: au moyen de la physique nous connaissons les êtres qui ont une existence matérielle dans la substance et dans la pensée; au moyen des mathématiques, les êtres qui ont une existence matérielle dans la substance et immatérielle dans la pensée; et, enfin, au moyen de la théologie, les êtres qui ont une existence immatérielle dans la substance et dans la pensée. Le parcours de la connaissance est graduel et il n'est pas possible de passer de la connaissance des choses matérielles à celle des choses immatérielles sans y transiter par la connaissance mathématique. Ici David établie une métaphore entre ceux qui veulent passer directement de la connaissance matérielle à celle immatérielle et ceux qui, tout en étant fermés dans un lieu brumeux, veulent regarder le soleil et deviennent aveugles. Ce métaphore nous rappelle le mythe de la Caverne de Platon, par lequel il décrit le parcours de la connaissance du philosophe, qui passe de la caverne au lieu illuminé par le soleil.

La philosophie se divise en théorie et en pratique comme l'entier se divise dans les parties d'une façon homogène, parce que la théorie et la pratique sont parties de la philosophie et quand une partie manque à l'entier, le tout entier est imparfait. On dit qu'elle se divise d'une façon homogène, parce que les parties se disent homonymes dans l'entier et les unes aux autres, comme la théorie et la pratique se disent philosophie, ainsi la théorique peut se dire pratique et vice versa. En outre, la philosophie se divise de l'un et vers l'un, parce que la physique, les mathématiques et la théologie sont nommées théoriques en tant qu'elles procèdent de l'unité de la théorie.

A son tour les mathématiques se partage en arithmétique, musique, géométrie et astronomie, en suivant la division de l'un et vers l'un, parce que toutes 
les quatre s'appellent mathématiques de l'unité des mathématiques. David

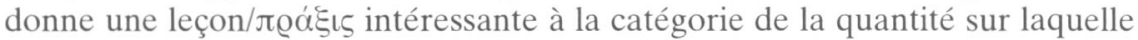
se fondent les quatre disciplines susmentionnées et, par la suite, la dédie aux inventeurs de ces disciplines, qui furent les Phéniciens pour l'arithmétique, les Thraces pour la musique, les Chaldéens pour l'astronomie et les Égyptiens pour la géométrie. L'importance donnée à la musique est considérable, étant donné que celle-ci peut avoir une fonctionne thérapeutique, parce qu'elle dispose l'âme aux différentes passions.

Ensuite, David traite de la division de la pratique, considérant que les Aristotéliciens et les Platoniciens ont des opinions divergentes sur cette question, puisque les Aristotéliciens la divisent en trois parties: éthique, économie et politique, tandis que les Platoniciens la divise seulement en deux parties: législative et la judiciaire. David essaie d'accorder l'opinion d'Aristote à celle de Platon, lorsqu'il dit:

„Et de même la ratio que porte la législative par rapport aux cités, en gardant la justice en elles, la judiciaire la porte par rapport à la législative, en gardant ce qu'elle ordonne, puisque l'éthique, l'économie et la politique se rapportent à la législative et à la judiciaire, comme leurs matières. En effet, le législateur ou bien orne-t-il les mœurs de quelqu'un ou bien orne-t-il la maison ou la cité, en donnant les lois selon lesquelles chacun doit se comporter"17.

Donc la tripartition d'Aristote n'est pas en désaccord avec la bipartition proposée par le Platoniciens, mais elle y est comprise et se manifeste dans la division platonicienne. La justice, en outre, est la vertu qui orne toutes les autres vertus de l'âme: le courage, le bon sens et la chasteté, parce qu'elle maîtrise les autres et maintient leur bon ordre. Le but de toutes les vertus est de brider le passions du corps et entre elles c'est surtout la justice qui fait connaître à l'homme que les voluptés corporelles générant des injustices.

La philosophie orne l'âme humaine et transforme la vie matérielle et brumeuse en vie immatérielle et divine, en particulier, au moyen de la philosophie théorique elle orne les facultés cognitives de l'âme, qui sont celles de l'intellect, de la réflexion, de la sensation, de l'opinion et de l'imagination, tandis que au moyen de la philosophie pratique elle orne les facultés animales, que sont celles de la volonté, de la décision, de l'intention, du désir, de la colère. Pourtant la philosophie est l'art réellement théorique, parce que, à la différence des autres arts, elle ne connaît pas seulement l'être matériel, mais plutôt tous les êtres et les choses divines, et on dit que la philosophie est la seule discipline réellement pratique, parce que les autres arts sont autour du corps, tandis qu'elle subsiste autour de l'âme.

${ }^{17}$ Dawit' Anyałt', Erkasirut'iwnk' p'ilisop'ayakank' [EEuvres philosophiques], éd. S. Arevšatyan, Erevan 1980, 101-102. 
III

Les questions liées à la figure et à l'œuvre de David touchent différents points: de nature philologique, mais aussi, et surtout, philosophique, bien que jusqu'ici les questions philologiques aient davantage retenu l'attention des chercheurs.

La première question concerne la langue, arménien ou grec, dans laquelle le texte original aurait été produit. Généralement on retient que les textes arméniens des traités sont des traductions de l'École hellénisante, traduits du grec comme plusieurs traités philosophiques des maîtres grecs (Aristote, Platon, Plotin, etc...). L'illustre savant Yakob Manandean, qui dédia un livre à la production de l'école hellénisante, pensait que David s'exprimât exclusivement en grec, tandis que un autre savant illustre, Francis Conybeare, considérait les Prolégomènes comme un texte écrit en arménien et le Commentaire à l'Isagoge comme un texte écrit en grec. Il y a sans doute une différence frappante entre les traductions des textes philosophiques grecs (par ex.: l'Isagoge de Porphyre, le De interpretatione et les Catégories d'Aristote) et celles des traités de David; cette différence est linguistique, parce que les premières sont souvent des reproductions presque servilement littérales des textes grecs, tandis que les deuxièmes présentent une expression linguistique dégagée du grec et quelque fois une différence textuelle non négligeable.

Donc il y a trois opinion, dont nous essayerons de donner une idée dans la schématisation suivante:

1) certains savants pensent que David s'exprimât en grec et que les textes grecs soient le résultat des notes prises par ses étudiants pendant les leçons à l'Académie d'Alexandrie, tandis que les textes arméniens seraient le résultat de l'activité de traduction de l'École hellénisante ${ }^{18}$;

2) certains savants pensent que David ait écrit certains œuvres en grec et d'autres en arménien ${ }^{19}$;

3) enfin, quelques autres savants pensent que David soit l'auteur des ses œuvres en arménien, tandis que les auteurs des versions grecques seraient ses élèves ${ }^{20}$.

18 Y. Manandean, Yunaban dproc'd yev ir zargac'man šrjannerd [L'École hellénisante et les phases de son développement], Vienna 1928; A. Topčyan, Remarks on David the Invincible's Commentary on Aristotle's "Prior Analytics", à paraître dans: Actes du colloque international sur „La diffusion de la pensée et des œuvres néoplatoniciennes dans la tradition arménienne et grécosyriaque. L'œuvre de David l'Invincible" (Université de Genève, 27-28 février 2004); B. Kendall R.W. Thomson, Definitions and Divisions of Philosophy by David the Invincibile Philosopher, Chico 1983 .

19 Cfr. S. Arevšatyan, Erker Dawit' Anhatt $i$ [Les œuvres de David l'Invincible], Erevan 1980; A. Terian, The Hellenizing School its Time, Place and Scope of Activities Reconsidered, dans: East of Bizantium: Syria and Armenia in the Formative Period (Dumbarton Oaks Symposium 1980), ed. N. Garsoian - Th. Mathews - R. Thomson, Washington 1982, 175-186. 
La question exige des études plus approfondies, surtout sur la langue hellénisante et, en particulier, sur la langue du Corpus davidien. Cependant, nous pouvons donner des exemples des différence textuelles - présentes dans les Prolégomènes à la philosophie - qui pourraient nous inciter à supposer soit un changement de la pensée de l'auteur depuis la rédaction de la version grecque à celle de la version arménienne, soit la possibilité que la version arménien puisse être la version originelle.

Dans le chapitre XIV des Définitions et Divisions de la philosophie, David cite plusieurs fois les nymphes ,éternelles”, tandis que dans la version grecque elles sont les nymphes qui vivent longtemps, mais non éternellement. Le passage en question traite du problème gnoséologique, puisqu'il s'interroge sur la possibilité de connaître la réalité au moyen de la définition. Dans la version grecque, David prend l'exemple de la définition de l'homme qui, comme animal, se distingue des non-animaux, comme raisonnable des irraisonnables, comme mortel des immortels et, enfin, comme réceptif d'intellect et de science des nymphes ,qui vivent longtemps" ${ }^{21}$. Par contre, dans la version arménienne il est dit que l'homme, comme animal, se distingue des non-animaux, comme raisonnable des irraisonnables, comme mortel des immortels et, comme réceptif d'intellect et de science des créatures immortelles qui s'appellent nymphes „éternelles”. La différance entre les deux textes est frappante, parce que dans le grec, les nymphes sont considérées des créatures mortelles, alors que, dans l'arménienne, elles sont considérées immortelles. Ici, la spécificité qui détermine la différance entre l'homme et les nymphes est dans le fait que le premier est réceptif d'intellect et de science et, au contraire, les nymphes sont douées de la science par nature,c'est-à-dire qu'elles ont une connaissance innée.

Il existe encore un autre aspect de la question. Dans la version grecque, nous lisons que non seulement l'homme est animal raisonnable, mais le sont aussi les nymphes „qui vivent longtemps"; donc cette version, dans ce contexte, ne parle pas des anges et des démons. Au contraire, la version arménienne affirme que l'homme est animal raisonnable de même que les anges et les démons. Il s'ensuit que dans cette version la mortalité n'est pas un attribut

${ }^{20}$ Cfr. H. Gabrielyan, Hay p'ilisop'ayakan mtk'i patmut'yun [L'histoire de la pensée philosophique arménienne], Erevan 1956.

${ }^{21}$ Nous avons recherché chez quels auteurs on y trouve les nymphes caractérisées de l'attribut "qui vivent longtemps" (makraiōnes). Le point auquel nos recherches sont abouties indique que cet attribut se trouve chez Olympiodore dans le Commentaire aux Meteorologica IV 301, 15-20: „En fait, Platon dit qu'il y a des animaux de feu et des animaux d'air, comme les nymphes qui vivent longtemps". Nous n'avons pas pu trouver le passage mentionné par Olympiodore. Chez Platon, les nymphes sont des êtres démoniaques, qui donnent l'inspiration divine aux hommes. Néanmoins il serait nécessaire de conduire des recherches plus approfondies sur la présence du mot «nymphe» chez les philosophes antérieurs à David. 
liée à la rationalité. Le domaine de ce dernier attribut comprend tant les hommes que les créatures angéliques et démoniaques ainsi que les nymphes qui sont aussi rationnelles et immortelles. Ce qui est en contradiction avec l'enseignement du texte grec. En ce domaine, le texte arménien s'aligne à la doctrine de Porphyre pour qui soit l'homme soit les dieux appartiennent au genre d',,animal”. Les dieux, ou le dieu, ne sont pas identifiés au Dieu-Un, mais plutôt aux divinités, comme les anges, les démons, les astres et les âmes ${ }^{22}$.

Dans le passage en question, il semble que le grec présente une contradiction, puisque si toutes les créatures angéliques et démoniaques sont des êtres immortels, alors les nymphes aussi devraient avoir la caractéristique de l'immortalité, en tant que créatures démoniaques et invisibles. Au contraire, elles sont considérées mortelles/qui vivent longtemps.

En outre, la question sur la langue originelle du Corpus Davidicum implique la problématique de la langue hellénisante et de la production en cette langue. Cette question aurait besoin d'un développement plus attentif que nous ne pouvons pas aborder maintenant. C'est important de souligner que les innovations grammaticales, lexicales et stylistiques, introduites par l'École hellénisante, donnèrent une contribution indéniable a l'enrichissement de la langue de l'époque maštoc'ienne. Cette nouvelle forme de la langue aurait aidé la compréhension et l'assimilation de la pensée philosophique et théologique du monde hellénistique, lequel aurait joué un rôle fondamental dans les discussions théologiques soutenues par l'Église arménienne avec l'Église byzantine à partir du VI ${ }^{\text {ème }}$ siècle. Le but des traducteur de l'École hellénisante a été le même qui a inspiré le créateur de l'alphabète arménien, c'est-à-dire un désir de transmettre à la nation arménienne la culture grecque et universelle pour lui donner des armes adaptes à sauvegarder et développer son identité ethnique ${ }^{23}$ et sa propre culture.

Enfin, en concluant ce bref exposé, nous ne pouvons que souhaiter que la pensée de David et la culture arménienne de l'époque postclassique, ou hellénisante, puisse être l'objet d'une attention et d'un intérêt croissants de la part d'arménisants, de philosophes et de philologues ${ }^{24}$.

${ }^{22}$ Cfr. Porphyrius, Isagoge 14, 1-5. D'ailleurs, nous voudrions remarquer que, dans le texte De Antro Nympharum, Porphyre considère les nymphes comme âmes en général.

23 Cfr. voir la note 2 pour les indications bibliographiques.

24 A ce propos nous voudrions signaler l'existence du projet international de recherche sur „La diffusion de la pensée néoplatonicienne en Arménie: l'œuvre du philosophe David l'Invincible", dirigé par Mme Valentina Calzolari dans le cadre du programme SCOPES du Fond National de la Recherche Scientifique. A Mme Calzolari et au Prof. Boghos Levon Zekiyan (Université Ca' Foscari de Venise) nous voudrions exprimer notre gratitude pour leur conseils et suggestions. 